
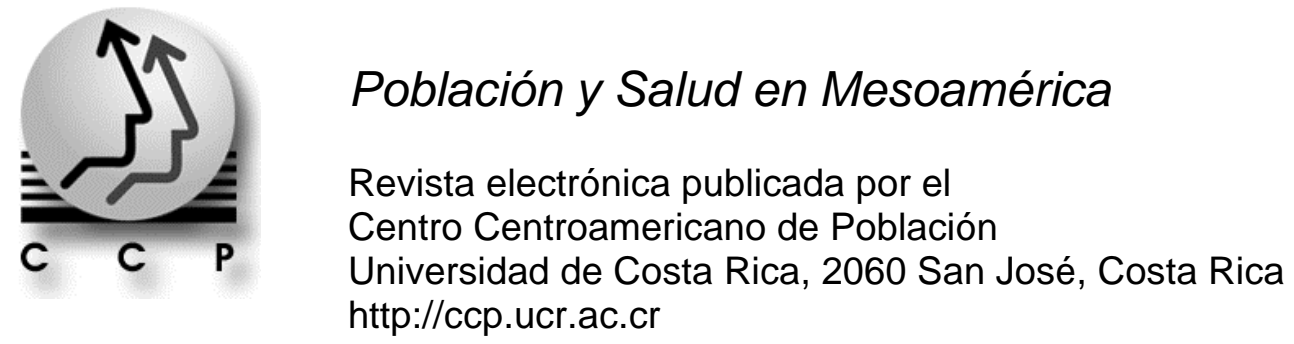

Población y Salud en Mesoamérica

Revista electrónica

Volumen 3, número 2, artículo 5

Enero - junio, 2006

Publicado 1 de enero, 2006

http://ccp.ucr.ac.cr/revista/

\title{
Etno-demografía de la étnia pech, Honduras
}

\section{Juan Carlos Vargas Aguilar}

(C) 2006 Centro Centroamericano de Población 


\section{Etno-demografía de la étnia pech, Honduras ${ }^{1}$}

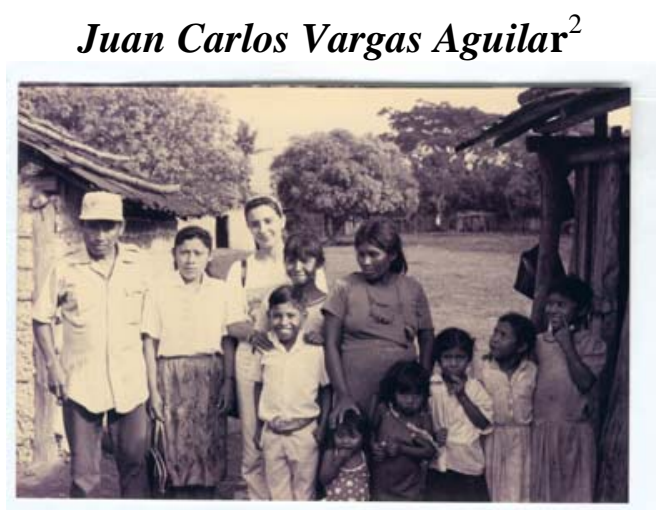

Familia pech-misquita (él pech, ella misquita) en Santa Maria del Carbón, Olancho.

\section{RESUMEN}

En este trabajo se discute la metodología y hallazgos de un estudio, bajo la modalidad etnodemográfica, de la étnia pech en el oriente de Honduras, diseminada en trece comunidades en los departamentos de Olancho, Colón y Gracias a Dios. Se realizó un censo de población como parte de una metodología aplicable a los diferentes grupos étnicos. La metodología empleada busca mostrar las posibilidades e importancia de relacionar variables demográfico-estadísticas con variables culturales bajo un análisis integrado logrando "relacionar las variables culturales con el comportamiento demográfico y las tendencias poblacionales" (Valdés, L.,1988). Se discuten también las diferentes preguntas para la determinación de la población étnica, en sus limitaciones y potencialidades. El trabajo de campo del estudio fue realizado bajo convenio de la Unidad de Docencia e Investigación en Población (UDIP) de la Universidad Nacional Autónoma de Honduras y el Instituto Hondureño de Antropología e Historia (IHAH) en 1994. Se emplean además algunos datos del censo de población más reciente en Honduras (2001), como punto de comparación y análisis del comportamiento demográfico de este grupo étnico. En los territorios de la etnia, en 1994 se contabilizaron 2079 personas (1020 hombres y 1059 mujeres), y para el último censo nacional (2001) la componían 3141 personas (1523 hombres y 1618 mujeres).

\section{ABSTRACT}

This work discusses the methodology of an ethno-demographic census on pech's ethnic group located in a valley at the Honduras' east. The objective of this study is to show the possibilities and the importance of relating demographic and statistical variables with cultural variables under an integrated analysis. It also discusses the different questions for the ethnic population's limitations and advantages. Field work was carried out in 1994, joined with the Teaching Unit and Research in Population (UDIP) of Universidad Nacional Autónoma of Honduras and the Honduran Institute of Anthropology and History (IHAH). It also used some data from the most recent population's census in Honduras (2001), as comparison for the analysis of this ethnic group demographic behavior. In the "pech" territories there where in 1994, 2079 people (1020 men and 1059 women). According to last national census data, the population grows to 3141 people (1523 men and 1618 women).

\footnotetext{
${ }^{1}$ El trabajo de campo se realizo en noviembre de 1994. La etnóloga Silvia González (tercera de izquierda a derecha en la foto) del Instituto Hondureño de Antropología e Historia (IHAH), participó activamente en la investigación de campo.

${ }^{2}$ Antropólogo Social, con especialidad en Demografía. En el momento del trabajo de campo laboraba como Especialista demógrafo del proyecto “Investigación, formación y divulgación en población” (HON/91/P05) del UNFPA en Honduras, organismo que cubrió los gastos de la investigación. jcvargas@ccp.ucr.ac.cr
} 


\section{INTRODUCCIÓN}

Peyser y Chackiel incluyeron a Honduras en el grupo de países con poblaciones indígenas relevantes a nivel nacional y que poseen zonas de resguardo o habitan en reducciones especialmente designadas y que su cultura no es tan fuerte ni generalizada debido, principalmente, a la limitación espacial (Peyser \& Chackiel, 1994). Pese a la importancia que han tenido las poblaciones étnicas, desde la Conquista y en la actualidad, se conoce poco sobre la dinámica de estas poblaciones.

Hasta 1988 diferentes censos en Honduras habían tratado el tema “indígena”, empleando básicamente dos definiciones: la fenotípica similarizada con raza y la lengua hablada. Pese a esto, los datos diseminados no permitían conocer a estos grupos mas allá de datos generales.

Por esta razón, y como ejercicio para poner a prueba un modelo metodológico que permitiera conocer y describir a las poblaciones étnicas en Honduras, en 1994 se desarrollo el trabajo de campo del Estudio etnodemografico de la etnia pech. Este trabajo lo realizo la Unidad de Docencia e Investigación en Población de la Universidad Nacional Autónoma de Honduras y en conjunto con el Instituto Nacional de Antropología e Historia.

En el ultimo censo de población, de 2001, se incluyo una pregunta sobre auto adscripción, que permitirá conocer en mayor profundidad la dinámica de los grupos étnicos en Honduras. Esto se dinamiza además porque se puede acceder a la base de datos mediante herramientas Internet, con lo cual se podrán realizar tabulaciones especificas para cada una de las poblaciones étnicas del país.

\section{ASPECTOS DE LA GEOGRAFÍA Y DEFINICIÓN TERRITORIAL}

Honduras se ubica en el norte de la región tropical, entre $12.58^{\circ}$ y $16.20^{\circ}$ de latitud norte y entre $83.10^{\circ}$ y $89.22^{\circ}$ de longitud oeste. Tiene un área de $112,492 \mathrm{~km}^{2}{ }^{3}$ y la más accidentada topografía de los siete países del Istmo Centroamericano. Comparte fronteras en el noroeste y oeste con Guatemala, en el suroeste con El Salvador y en el este con Nicaragua. En el norte, Honduras tiene una región costera estrecha frente al Mar Caribe que se extiende por unos $650 \mathrm{Km}$. Su región costera en el sur es más pequeña, siendo restringida enteramente a las costas del Golfo de Fonseca, que se extiende por unos $120 \mathrm{Km}$. entre las costas de El Salvador y Nicaragua.

Su topografía es altamente accidentada producto de las dieciséis cordilleras, ubicadas entre las planicies costeras del Mar Pacífico y del Mar Caribe, y que hacen el 75\% del terreno tenga pendientes que exceden el 25\% de gradiente (PAH, 1989). Muchos de los eventos geológicos antiguos de la región, así como las condiciones climáticas, ejercen una influencia importante sobre los ecosistemas, el uso de los recursos naturales y la distribución espacial de la población en Honduras.

\footnotetext{
${ }^{3}$ Dato actualizado y oficializado el 12 de septiembre de 1994, luego del veredicto de la Corte
} Internacional de La Haya, en el litigio con El Salvador por distintos territorios. 
Las tierras bajas de Honduras, que se encuentran en las zonas costeras, así como en los veinte pequeños valles, constituyen los mejores suelos, pero no ocupan más de $6.2 \%$ del territorio. Estos llanos costeros penetran al interior en la vertiente del Caribe hasta los valles de los Ríos Ulúa, Chamelecón y Aguán. En el este del país, los Ríos Patuca y Segovia han formado una planicie aluvial amplia, cuya extensión excede todas las otras regiones bajas combinadas. Esta región baja, generalmente conocida como La Mosquitia, pasa gradualmente a elevaciones mayores al suroeste en las partes orientales del Departamento de Olancho, zona de asentamiento ancestral de la población pech.

Los muchos ríos que tienen su origen en este territorio montañoso han tenido impacto en el desarrollo de las comunidades del país, permitiendo el riego de cultivos y facilitando la comunicación y el transporte donde los caminos son pobres o inexistentes. También los ríos tienen crecidas periódicas que pueden destruir la producción e infraestructura de las comunidades aledañas a ellos.

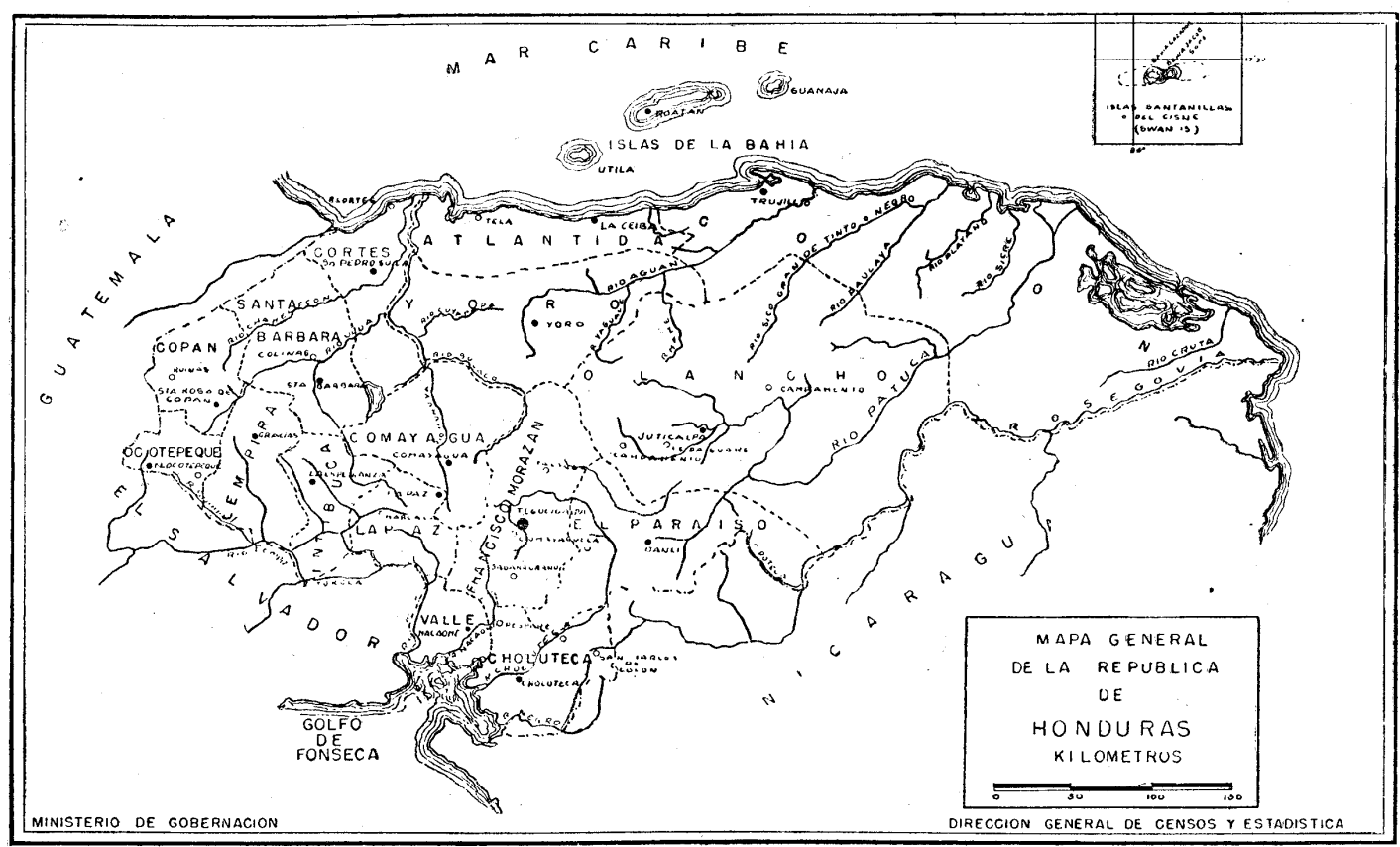

\section{LAS POBLACIONES PRE-COLOMBINAS}

La historia demográfica de Centroamérica en general es poco conocida y Honduras presenta en sí un mayor enigma. Con relación a la población de Centroamérica (y Honduras particularmente), la controversia respecto a la población al momento de la Conquista es grande y no existe acuerdo entre los investigadores, pues no ha sido posible determinarla con exactitud. Se pasa de las estimaciones de Kroeber (1939), de 100,000 pobladores para Honduras y Nicaragua, hasta las de Dobyns (1966) de 10.8-13.5 millones para Centroamérica en conjunto. Varias referencias indican que en el período de la Conquista se dieron gran cantidad de muertes, lo cual sugiere debió existir una importante población indígena 
original. Como estas estimaciones son para el momento de la Conquista, aclaran poco sobre la dinámica poblacional de los pueblos involucrados. Sapper (1924) realizó una estimación para Centroamérica de seis millones de habitantes, en la que tomó en cuenta el clima, tecnología y recursos, lo cual la convierte en una aproximación aceptable.

La falta o poca investigación científica de los muchos sitios arqueológicos en Honduras limitan que esta fuente pueda ser utilizada para desvelar la población en esos períodos. Esto hace las fuentes documentales las utilizadas primordialmente. Para ello debe remitirse la investigación a los cronistas contemporáneos, con las dificultades que ello entraña ${ }^{4}$. El único relato de un cronista contemporáneo (1541) indica una población de 400,000 personas para Honduras, al momento de la Conquista (Benzoni,1967:163), cifra que Newson (1992a) considera baja. Esta autora, basándose en evidencia cultural y ecológica, estima la población pre-colombina de Honduras en más de un millón, como se muestra en el Cuadro 1.

\section{Cuadro 1. Honduras: Estimados de Poblacion indígena antes de la conquista basados en evidencia cultural y ecológica}

\begin{tabular}{lccc}
\hline & $\begin{array}{c}\text { Area } \\
\text { en km }\end{array}$ & $\begin{array}{c}\text { Densidad } \\
\text { estimada }\end{array}$ & $\begin{array}{c}\text { Población } \\
\text { estimada }\end{array}$ \\
Occidente y Centro & & & \\
Oriente & 42,563 & 20 & 851,260 \\
Area Xicaque $^{\mathrm{c}}$ & 52,897 & 10 & 528,970 \\
Total & 16,628 & 1 & 16,628 \\
& 112,088 & 12,5 & $1,396,858$ \\
a/Departamentos: Cortés, Santa Bárbara, Copán, Ocotepeque, Lempira, Intibucá, Comayagua, La \\
Paz, Francisco Morazán, Valle y Choluteca. \\
b/Departamentos: El Paraíso, Olancho, Gracias a Dios, Islas de la Bahía y este de Colón. \\
c/Departamentos: Atlántida, Yoro y oeste de Colón.
\end{tabular}

Fuente: Newson, 1992a: 118, Cuadro 1

La composición de la población de Honduras poco antes del descubrimiento también no se conoce en detalle, aunque hay cierto consenso de que ésta se dividía en dos grupos culturales con características bien distintas y en diferentes fases de evolución social. El primer grupo, ubicado en el occidente y el sur de Honduras y en las zonas cerca de Trujillo y Juticalpa y las Islas de la Bahía, vivía en ciudades permanentes, tenía una agricultura bien

\footnotetext{
${ }^{4}$ La importancia que tenían para efectos del tributo y prebendas obtenidas de la Corona hacen que en los relatos e informes oficiales se dedicara especial atención a datos poblacionales, lo cual confiere a estas fuentes un valor importante para comprender la dinámica poblacional. No obstante, debe tenerse mucho cuidado en su análisis, pues no siempre se obtuvieron en forma sistemática ni con periodicidad. Además, están matizadas de la visión particular de los conquistadores y en algunos casos de su necesidad de "justificar" ante la Corona su poco tributo o bien "aumentando" la población poder obtener mayores concesiones.
} 
desarrollada basada en el maíz, un sistema nítido de diferenciación social y sistemas religiosos bien organizados, con templos, sacerdotes y ceremonias consolidadas. El segundo grupo, ubicado en la costa norte y la zona nororiental, probablemente era seminómada, vivía en casas comunales, dentro de aldeas pequeñas, con poca diferenciación social y dependía de los recursos salvajes, como la caza, con una agricultura incipiente basada en yuca, camote, malanga y otras raíces.

El primer grupo generalmente se denomina "mesoamericano" y pertenecía a familias lingüísticas diferentes: el grupo Maya-Quiché (chortí, chontal), Uto-Azteca (pipil, nicarao y mexica), Lenca y Oto-Mangue (chorotega). El segundo grupo, que generalmente se denomina "de bosques húmedos" o "de la zona intermedia" engloba dos subgrupos lingüísticos: Hoka-Sioux (tolupán o xicaque) y Macro-Chibcha (paya o pech, sumu y matagalpa). Algunos de estos idiomas eran hablados por varias tribus lenca (los cares, cerquines, potones y lencas propiamente dicho), el sumu (ulúas, panamakas y tawahkas) y matagalpa (pantasmas y parrakas).

No hay evidencias de la existencia de esclavitud o trabajo forzado entre las tribus de los bosques húmedos y tampoco entre los lencas, aunque estas instituciones eran comunes entre las demás etnias centroamericanas. Este es uno de los ejemplos de rasgos culturales por los cuales los lencas parecen haber ocupado una posición intermedia entre los pueblos mesoamericanos y los de los bosques húmedos.

Es poco lo que se conoce respecto del despoblamiento de la región que fue el producto de la conquista. El conjunto de varios factores, no siempre mencionados en la literatura, brinda los elementos para explicar y entender el declive de la población , tal como se explica a continuación:

Conquista: Este proceso fue especialmente cruel en la región centroamericana. Basta recordar las incursiones de Pedro de Alvarado. La resistencia indígena jugó papel fundamental en esta situación, por lo que la Conquista se realizó muy cercana a la espada y menos a la cruz. Lempira se enfrentó con un ejército compuesto por al menos veinte mil hombres, lo cual muestra la población envuelta en el proceso. Una vez realizada la Conquista, la población indígena se vio fuertemente diezmada. Benzoni (1967) indica una población de 8,000 indígenas para 1541 y el Obispo Pedraza indica 15,000 indígenas para 1539 (citado en Newson, 1992 a: 177), números probablemente subestimados. Griffin (1992) estima que en 1544 la población indígena había sido reducida a $25 \%$ de su tamaño original, mientras Newson (1992 a) estima que en 1575 este porcentaje ya se aproximaba al 5\% del original.

Esclavitud: Como Honduras inicialmente no parecía poseer productos valiosos de exportación, el interés de los españoles se concentraba en la captura de esclavos para sus otras colonias. Directamente de Honduras se llevaron cerca de 150,000 indígenas, principalmente de la zona de Trujillo, para trabajar en Cuba, Jamaica y Hispaniola antes de 1548. Al comienzo, cuando había una superabundancia de esclavos, los vendían a 2 pesos cada uno. Cuando se tornaron más escasos, el precio subió. Ya en la década de los treinta era de 6-7 pesos y en la década de los cuarenta 50-60 pesos, lo que todavía era barato en comparación con el precio que se pagaba en las otras 
colonias. Después que encontraron minas en Honduras, los españoles siempre se quejaban de la falta de mano de obra que se había provocado en parte debido a esta práctica (Griffin, 1992).

Maltrato y sobretrabajo: Los tipos de trabajo a los que fueron sometidos y mortalidades que ello acarreaba llevaron a prohibir que se utilizara población indígena en esos trabajos (siendo sustituida por población esclava de origen africano). Esto desencadenó en las "leyes de indias", entre otros por la presión ejercida por Fray Bartolomé de las Casas. Estas "nuevas leyes", que impedían la esclavitud y otros malos tratos a la población aborigen, fueron introducidas efectivamente en 1550, por lo que para efectos de América Central tuvieron poco efecto, puesto que la Conquista, con toda su crueldad, ya se había completado. Las nuevas leyes sí agravaron la falta de mano de obra para la explotación de las minas que inicialmente se había creado por la exportación de esclavos.

Enfermedades: Según Newson (1992a), el papel de este factor en el despoblamiento se ha sobrevalorado. Si bien es cierto que impactó en la morbilidad y mortalidad de la población aborigen, debe tomarse en cuenta que su influencia fue diferencial según la zona y grupos involucrados (fundamentalmente la distinción entre los pueblos de la costa y las sierras). Así mismo, la evidencia reciente indica que su participación impactó más al final de la Conquista y sobre todo en la Colonia. Se ha indicado y documentado que las primeras epidemias de viruela ocurrieron en México. Debe tenerse en cuenta que este factor no actuó sólo y posiblemente se diera en conjunción con el maltrato y sobreexplotación de la fuerza de trabajo. Por otro lado, MacLeod (1973) estima que, aun antes que la Conquista de Centroamérica comenzara en serio, las epidemias de peste y viruela de 1519-1520 ya habían matado a un tercio de la población indígena de la región.

Desestructuración económica-social: Si bien las enfermedades jugaron un importante papel, se ha minimizado o poco tomado en cuenta la destrucción social/económica como elemento importante en este proceso. Cambios producidos en la estructura cultural en cuanto a producción, tecnología, así como las formas organizativas de la sociedad afectaron fuertemente la sobrevivencia de los grupos indígenas, llevando a la desnutrición y hasta la muerte. Esto al ser reducidas sus tierras y alteradas sus formas productivas, sobre todo por las demandas de tributos y obligatoriedad de utilización de la fuerza de trabajo en otras labores que les limitaban dedicarse a su producción.

El conjunto de estos elementos posiblemente alteró la relación de sexos y con ello la tasas de natalidad. Newson (1981) ha sugerido, con base en diferentes fuentes, que estas situaciones resultaron en prácticas de control natal por parte de la población indígena y que el tamaño típico de las familias indígenas no pasaba de uno o dos hijos. La separación de sexos, por el tipo de trabajo, llevó a la abstinencia sexual. También se practicó el aborto inducido, así como el infanticidio. Con base a los datos expuestos por esta autora, puede argumentarse que el despoblamiento o reducción de la población indígena en Honduras en el siglo XVI llevó a la imposibilidad de su recuperación en el siglo XVII. La Conquista fue particularmente destructiva en Honduras. Se calcula una razón de 24:1 para la despoblación del área colonizada y de la mitad o una tercera parte en la zona no colonizada. Es poca la investigación que se ha realizado en este sentido y en sí las fuentes disponibles son escasas $\mathrm{y}$ fragmentarias. 


\section{EL PERÍODO COLONIAL (1500-1821)}

Como se indicó, Honduras tiene escasa información analizada para el siglo XVI, situación que se repite para el período colonial. Ello es similar para los demás países de la región, en la cual se han explorado poco las fuentes históricas desde la perspectiva demográfica. De esta forma, se encuentra disponible y urgido de investigación el campo de la demografía histórica, que será de importancia para comprender la dinámica de la población hondureña.

Es notable que la penetración española al interior de Honduras tardó en concretarse. Chamberlain (1966) observa que los españoles de Trujillo tenían el mismo número de soldados que Pizarro usó para subyugar todo el Perú, pero nunca llegaron a conquistar el interior. En 1544, veinte años después que la Conquista comenzó en serio, los españoles tenían un control precario sobre la región de Puerto Cortés (entonces Puerto Caballos) a Comayagua y de Comayagua a Guatemala. Entre Puerto Cortés y Trujillo, de Comayagua hacia el sur y del Río Plátano hacia el este prácticamente no tenían control. De Comayagua hacia Olancho controlaban algunos pueblos, pero de Catacamas a Trujillo solamente controlaban un camino real. El actual Departamento de El Paraíso estaba igualmente fuera de su control (Griffin, 1992). En la década de los setenta se incorporaron Tegucigalpa y Choluteca. Sin embargo, aún en 1700, el actual Departamento de Atlántida, la parte occidental de Yoro, el norte de Olancho, la frontera de Olancho con El Paraíso y todo el Departamento de Gracias a Dios, con las partes vecinas de Colón, Olancho y El Paraíso (una zona llamada Taguzgalpa en la época) todavía estaban fuera del dominio español, situación esta que cambió relativamente poco durante todo el siglo XVIII. Además de factores climáticos y topográficos, la propia organización social de los indígenas de los bosques húmedos en estas zonas, con sus aldeas pequeñas de 150-250 habitantes, su existencia semi-nómada, su falta de una autoridad central y su falta de tradición con el trabajo forzado y el que debieran pagar tributo dificultaban su incorporación. Griffin (1992).

Durante el siglo XVII dos nuevas etnias aparecen en Honduras: los misquitos y los garífunas. Según cuenta la historia, el origen de los mosquitos está en el naufragio de un barco portugués en 1641, cerca del Cabo Gracias a Dios (Mariñas Otero, 1987). Los esclavos africanos sobrevivientes se unieron a las tribus sumu de la región, formando una nueva cultura con rasgos híbridos. Parece que las sociedades tribales originales de Honduras y Nicaragua eran predominantemente monógamas, pero la poliginia entre los misquitos era muy común. Muchas de las mujeres eran robadas de otras tribus, lo que favorecía el crecimiento demográfico de este grupo y no así los demás. Otro mecanismo que afectó al crecimiento de las demás tribus fue que los misquitos se convirtieron en guerreros especializados en el tráfico de esclavos para la exportación. A diferencia de las otras tribus, los misquitos también estaban organizados en un estado nacional, con una dinastía de reyes hereditarios, lo que facilitó los contactos con los europeos, principalmente los ingleses, que llegaron a aceptar la Mosquitia como un protectorado inglés (Griffin, 1992).

El censo de 1801 enumeró un total de 1,512 familias españolas y 13,553 familias ladinas (un total de 92,971 personas para el conjunto de los dos grupos), 8,298 indígenas que pagaban tributo y 27,184 fuera del control de los españoles. Sin embargo, el gobernador de Honduras estimó en 1804 que había 16,000 tolupanes entre Omoa y Gracias a Dios,

http://ccp.ucr.ac.cr/revista/ 
15,000-30,000 misquitos al este del Río Tinto y 10,000-12,000 pech y tawahkas fuera de su control (Griffin, 1992).

\section{EL PERÍODO MODERNO (1821-1950)}

En esta época, Honduras presentaba una economía descentralizada, para lo cual se pretendió la construcción del ferrocarril interoceánico y poder desarrollar la producción agropecuaria, proyecto que fracasó. El ferrocarril tan sólo se concretó para la zona norte y unas 110 millas de la costa. Fue utilizado para el transporte de la producción bananera.

En 1838, el Arzobispo de Guatemala, Don Francisco de Paula García Peláez, calculó la población de Honduras en 173,365 habitantes: 21,165 españoles, 106,668 ladinos y 45,532 indios (Mariñas Otero, 1963). En 1855, Squier dio una estimación alternativa de 350,000, entre blancos $(50,000)$, ladinos $(60,000)$, indios $(100,000)$ y negros y mulatos (Squier, 1856; Wells, 1982). No se sabe cuál fue el criterio usado para distinguir entre indios y ladinos y es casi cierto que el total está sobreestimado. El censo de 1887, que enumeró una población de 331,917, clasificó a 68,872 individuos como indígenas, o sea, aproximadamente 21\% del total. En 1930, este número era de 85,769 (10 \%) y en 1945 se había reducido a $80,660(6.6 \%)$.

Es a partir de la segunda mitad del siglo XIX que los indígenas gradualmente pierden el dominio sobre sus tierras y que se incorporan a la economía nacional los territorios como Atlántida y parte de Yoro, que todavía estaban bajo control indígena. Este proceso se dio principalmente a través de las concesiones dadas a hondureños y extranjeros por el gobierno central. Los tolupanes y pech perdieron sus tierras en la zona de Tela y las regiones de Río Sico y Río Paulaya con la “Tela \& Truxillo Railroad Company”. También se dieron concesiones para cortar madera, particularmente caoba, en las zonas de los pech, misquito y tawahkas, además de concesiones para hule, ganado, corozo, oro, carbón, cocos, etc. Como los indígenas de la zona nororiental nunca habían estado bajo la jurisdicción efectiva de los españoles, no había tierra ejidal en esta región y prácticamente toda la tierra era nacional En algunos casos los indígenas huyeron más al interior; en otras, se integraron por periodos trabajando en las nuevas empresas, pero esta mano de obra no era suficiente y segura, por lo que los concesionarios tenían permiso para importar trabajadores extranjeros. Llevaron trabajadores del centro del país, de El Salvador, de Belice, las Islas Caribeñas, de Europa y del Medio Oriente. No les fue permitido traer negros o chinos, pero a veces lo hicieron clandestinamente, particularmente la Truxillo Railroad Company. Cada uno de los inmigrantes legales tenía derecho hasta 7 caballerías de tierra y el gobierno hondureño apoyó la publicación de libros en varios idiomas europeos para ayudar a reclutar trabajadores extranjeros. Podían recibir su ciudadanía más rápidamente si se asentaban en tierra indígena (Griffin, 1992; Kepner, 1936; Tojiera, 1982).

El Censo de 1881, que contó 307,289 habitantes, tuvo un porcentaje muy alto de subenumeración debido a la oposición de los indígenas a ser contados (Goyer \& Domschke, 1983: 215). La Dirección General de Estadística Nacional presentó tabulados resumen conteniendo la siguiente información (Vallejo, 1893; DGEC, 1981): 
- Número de casas y edificios según departamento;

- Población por grupos de edad según sexo y departamento;

- Población clasificada según profesiones y oficios;

- Población según la educación (sabe leer, sabe escribir), el estado civil (soltero, casado, viudo), la capacidad política (ciudadanos electores, ciudadanos elegibles y empleados públicos) e inmigración (migrante, no migrante).

\section{POBLACIONES ÉTNICAS EN LA ACTUALIDAD}

Honduras cuenta en la actualidad con seis minorías étnicas diferenciadas, a saber: garífunas, lencas, xicaques o tolupanes, pech o payas, misquitos y tawahkas. Sobre la dinámica y el volumen de la población de estos grupos es poco lo que se conoce. En general, los estudios existentes se refieren a aspectos de orden antropológico-etnológico y/o bien sobre la situación socio-económica de privación en que viven. La información socio-demográfica es limitada y, cuando se encuentra, se trata de datos aislados, utilizados en documentos que buscan dar cuenta de la situación social más general, empleando algunos datos demográficos para dar un panorama global sin profundizar en el análisis de la situación demográfica en sí.

De las fuentes de información existentes, a nivel agregado, se tiene la proporcionada por el Censo de Población y Vivienda de 1988. En éste se introdujo una pregunta que apunta en alguna medida en la variable étnica. La pregunta fue:

"Habla usted alguna de las siguientes lenguas: Garifuna, Xicaque, Paya, Misquito, Lenca, Sumo o ninguna de las antes mencionadas".

Esta pregunta, aunque subestima la población, posibilita - en forma rudimentaria - acercarse al conocimiento de su volumen. Esta subestimación se da por la definición misma, al tomar solamente a los mayores de 5 años y por su “estructura” pues solo contabiliza aquellos que declaran emplear la lengua o idioma. Además, la forma en que se realizo solamente capturo una lengua hablada, por lo que no se conoce sobre el multilingüismo. Por otro lado, esta pregunta posibilita tomar en cuenta la población perteneciente al grupo étnico sin restringirla a zonas definidas.

A partir de los resultados obtenidos con esta pregunta, Ponce (1993) realizó un trabajo sobre las características poblacionales y socio-económicas (más particularmente educativas) de estas poblaciones, encontrando 23,195 misquitos, 22,020 garífunas, 1,555 tolupanes, 925 pech, 779 lencas y 315 tawahkas. Este estudio debe tenerse con reservas en el sentido de que la pregunta se refería a si la persona hablaba o no alguna de las lenguas indígenas. Ello no es sinónimo de que la persona pertenezca al grupo étnico de esta lengua. El criterio es particularmente inapropiado en el caso del grupo lenca, cuyo idioma desapareció ya en el siglo XIX. El hecho de que aun así el censo encontró 779 hablantes del idioma ya es una indicación de la precariedad de la información. Por otro lado, la pregunta se aplicó sólo a personas mayores de 5 años, con lo cual debe de estimarse la población menor de esa edad. Otro elemento que debe tenerse en cuenta, al evaluar los datos aportados por el censo a partir de esta pregunta, es lo concerniente a la subenumeración. 
Las poblaciones indígenas y garífunas a las cuales se dirige la pregunta se encuentran en zonas de menor accesibilidad y por ello son susceptibles de tener una subenumeración mayor, respecto de otros grupos poblacionales. Se desconoce en realidad la extensión que esta situación haya podido alcanzar, puesto que no se realizaron estudios específicos de evaluación postcensal para medirla.

Por otro lado, Tamayo (1994), utilizando el mismo censo, realizó un acercamiento a la dinámica de población del grupo tawahka. El esfuerzo realizado mostró la inconveniencia y limitación que esta fuente presenta para el estudio de poblaciones particulares. Sobre todo la limitación en este caso se encuentra en que el censo de 1988 no buscó realizar un diferenciación clara de la población indígena (y étnica en general), por lo cual no se puede pretender que brinde información que posibilite conocer en profundidad las características socio poblacionales de estos grupos. A su vez, para aprehender la realidad poblacional de pequeños grupos, no es posible usar algunas de las técnicas demográficas tradicionales, que permiten un adecuado acercamiento a grandes grupos, por la variabilidad de las cifras y la poca eficiencia estadística de los métodos.

Rivas en 1993, obtuvo la cifra de 2585 personas habitando en los poblados pech. Sus datos los obtuvo para el total de la población (sin desagregar por edad y sexo ni otras características) mediante consulta directa a líderes de las comunidades.

En noviembre de 1994, se llevo a cabo el trabajo de campo para la recolección de los datos del censo etnodemográfico de la etnia pech. Este se realizó como un proyecto conjunto entre el Instituto Hondureño de Antropología e Historia (IHAH) y la Unidad de Docencia e Investigación en Población (UDIP) de la Facultad de Economía, Universidad Nacional Autónoma de Honduras (UNAH) y como un proyecto piloto para poner a prueba una metodología que permitiera acercarse al comportamiento socio-demográfico de los grupos étnicos del país. La población censada en los 14 poblados pech alcanzo 2079 personas, 1020 hombres y 1059 mujeres para un índice de masculinidad de 0.96. El criterio fundamental de definición étnica en este estudio estuvo en la residencia en los pueblos del grupo étnico, aunque también se tomo en cuenta la lengua hablada. Los datos de este estudio se presentan mas adelante.

En el censo de población del 2001, se empleo la auto adscripción como criterio para determinar las poblaciones étnicas en Honduras. A partir de esta pregunta se reportaron 3848 personas pertenecientes al grupo étnico pech en todo el país, según se aprecia en el grafico 1. 


\section{Gráfico 1. Honduras: distribución de la población pech en el territorio. Censo de población, 2001.}

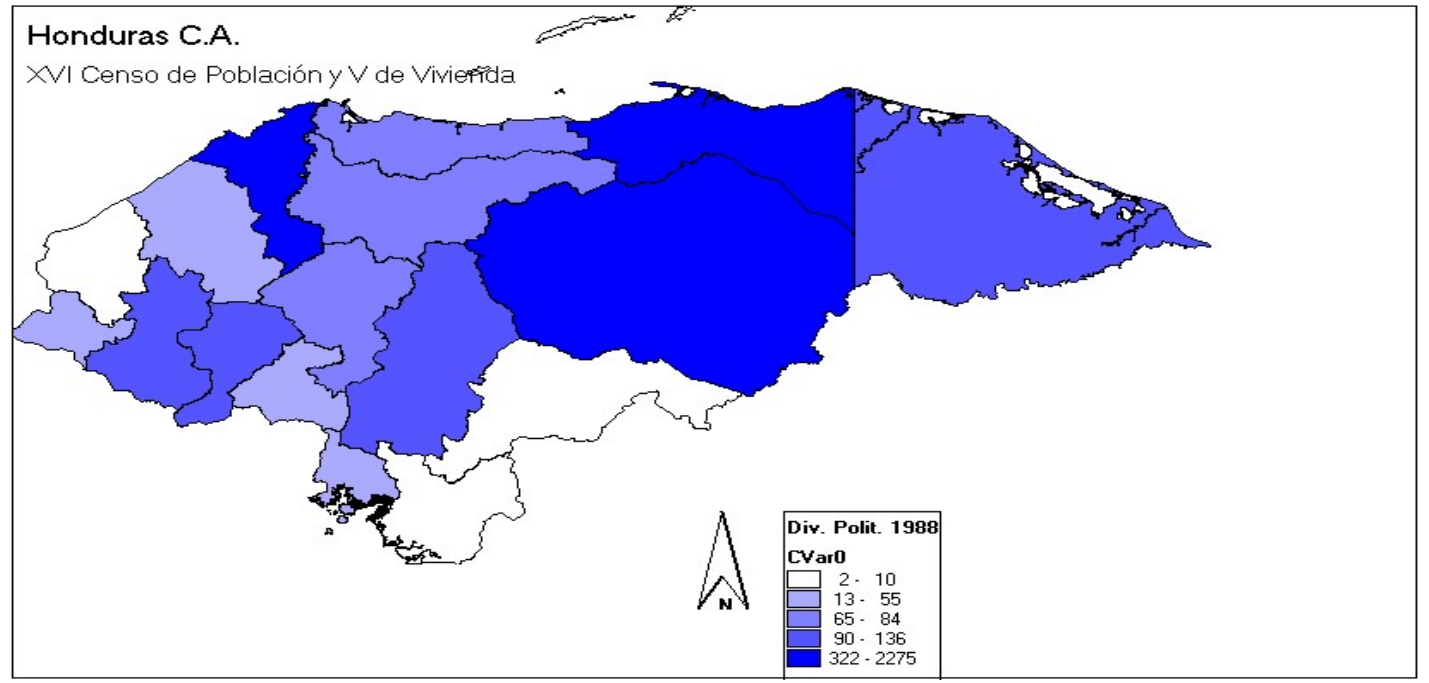

Fuente: http://www.ine.hn.org

En la mayoría de los departamentos de Honduras se reporto la presencia de población pech, aunque es notoria la concentración que se da en los departamentos Olancho y Colon y en menor medida en el departamento Gracias a Dios, zonas en las que se encuentran los “territorios pech".

Importante presencia de población de este grupo étnico se muestra también en los departamentos de Puerto Cortes (San Pedro Sula) y de Francisco Morazán (Tegucigalpa), las dos ciudades principales del país. Así como en departamento de importante presencia de otros grupos étnicos: Lempira e Intibucá. Para los territorios pech (los mismos del estudio de 1994) se reportaron 3141 personas.

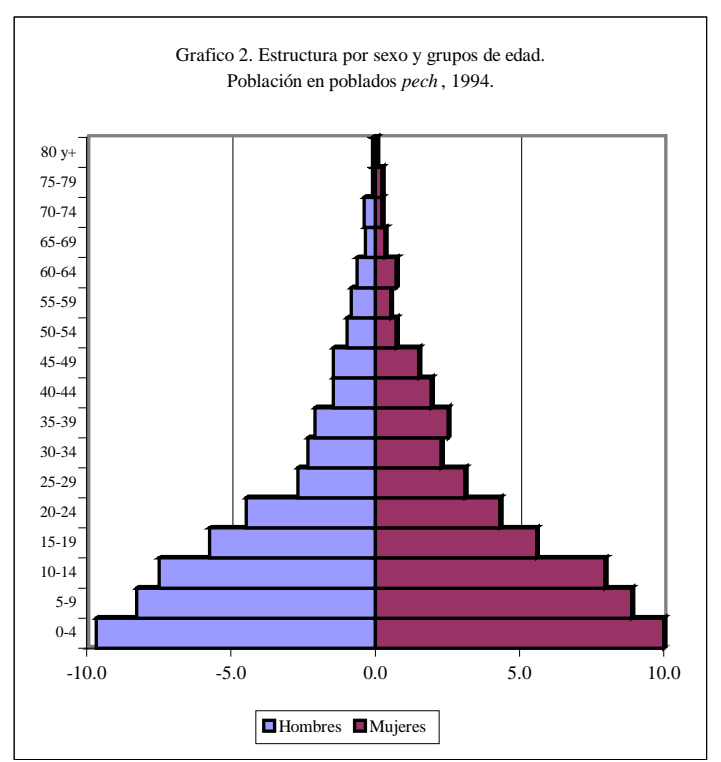

Fuente: Censo Pech, 1994

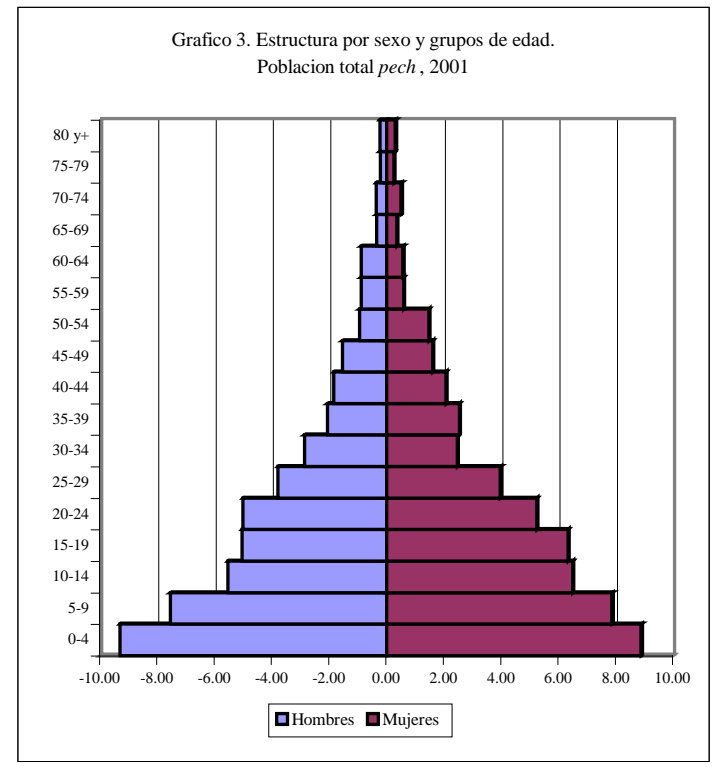

Fuente: Censo de Población 2001. [http://www.ine.hn.org] 
Los gráficos 3 y 4 muestran la estructura de la población pech para dos periodos. En 1994, se refiere a población en los poblados pech y el criterio empleado es sobre la base del lugar que habita por lo que se refiere a la población en los territorios pech. En el 2001 se empleo como criterio la "autodefinición”, por lo que en el gráfico se muestra la población en todo el país que se definió como perteneciente a esa etnia.

Se muestra que la estructura ha variado poco, con una leve reducción de la base y aumento de la población adulta mayor (de 60 y más años) . La relación entre sexos es muy similar, dándose en el periodo una leve reducción de la cantidad de hombres (cuadro 2), que puede ser atribuible al criterio empleado para la definición de la población. En el grafico 3 se mostró la distribución geográfica de esta población.

La población pech muestra un importante crecimiento, sea que se trate de la población total o bien de la población en sus territorios (cuadro 2). En el periodo 1994-2001 la tasa de crecimiento alcanzo 5.9 lo que indicaría que su duplicación (de persistir estas condiciones) se daría para el año 2012.

La relación entre los sexos se ha mantenido muy similar, especialmente entre las dos últimas fuentes. Como la definición empleada en el censo de 1988 es sobre la lengua hablada, y esta se “ perpetua” con mayor fuerza en las mujeres, ello explica -al menos en parte- la diferencia del índice de masculinidad con los de 1994 y 2001.

\section{Cuadro 2. Población total y sexo, índice de masculinidad y tasa de crecimiento según información población en los territorios pech. Censos de 1988, 1994, 2001}

\begin{tabular}{lrrrcc}
$\begin{array}{l}\text { Fecha } \\
\text { censo }\end{array}$ & Total & Hombres & Mujeres & $\begin{array}{c}\text { Índice de } \\
\text { Masculinidad }\end{array}$ & $\begin{array}{c}\text { Tasa de } \\
\text { crecimiento }\end{array}$ \\
\hline $1988^{\prime *}$ & 798 & 378 & 420 & 90.0 & -- \\
1994 & 2079 & 1020 & 1059 & 96.3 & 15.9 \\
$2001^{1 * *}$ & 3141 & 1523 & 1618 & 94.1 & 5.9 \\
\hline $\begin{array}{l}\mu^{* * *} \text { Estimada para los territorios pech, a partir de la población de 5 años y más del censo. } \\
\text { Funtimada para los territorios pech }\end{array}$ \\
Fuentes: INE-Honduras. Censos de Población. UDIP. Censo Etnodemografico pech
\end{tabular}




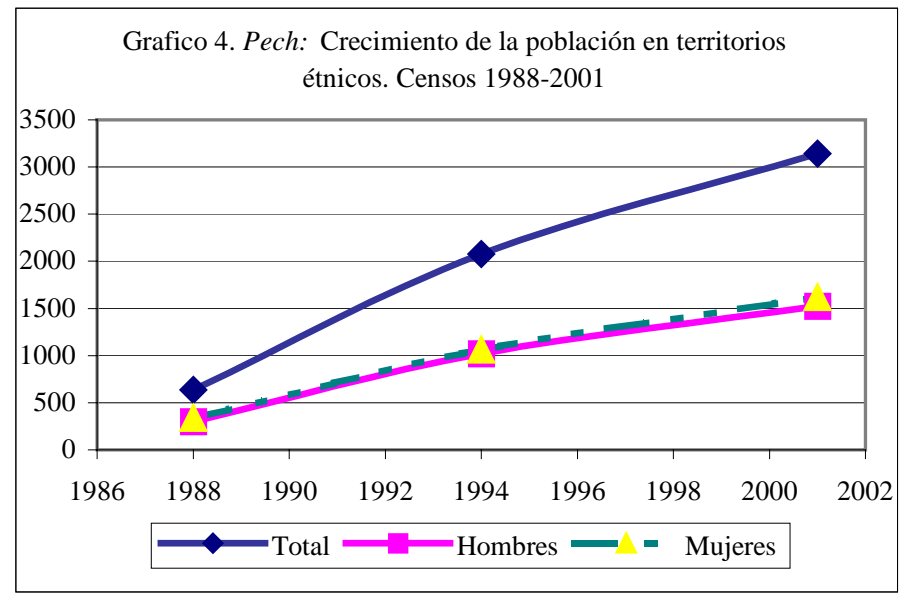

Cerca del $80 \%$ de la población total pech se encuentra en sus territorios (cuadro 3), aunque este porcentaje alcanza solamente el $66 \%$ de los jefes, lo que unido a los datos del grafico 2 , se muestra una alta dispersión de esta población que hace insuficiente el criterio de residencia en los territorios para conocer y dar cuenta de este grupo étnico.

\section{Cuadro 3. Honduras: Población pech según lugar de nacimiento y sexo por parentesco. 2001}

\begin{tabular}{rrrrrrrrrr} 
& \multicolumn{3}{c}{ Masculino } & \multicolumn{3}{c}{ Femenino } & & & Total \\
\cline { 2 - 9 } Nació aquí / Parentesco & Si & No & Total & Si & No & Total & Si & No & Total \\
\hline Jefe o Jefa del hogar & 375 & 173 & 548 & 60 & 47 & 107 & 435 & 220 & 655 \\
Cónyuge o pareja & 3 & 8 & 11 & 357 & 150 & 507 & 360 & 158 & 518 \\
Hijo(a) o hijastro & 883 & 105 & 988 & 868 & 90 & 958 & 1751 & 195 & 1946 \\
Yerno o nuera & 31 & 3 & 34 & 53 & 8 & 61 & 84 & 11 & 95 \\
Nieto(a) & 133 & 9 & 142 & 141 & 8 & 149 & 274 & 17 & 291 \\
Padre, madre o suegra & 7 & 2 & 9 & 27 & 11 & 38 & 34 & 13 & 47 \\
Hermano(a) o cuñado & 29 & 12 & 41 & 40 & 17 & 57 & 69 & 29 & 98 \\
Sobrino(a) & 30 & 6 & 36 & 35 & 5 & 40 & 65 & 11 & 76 \\
Otro Pariente & 22 & 6 & 28 & 27 & 16 & 43 & 49 & 22 & 71 \\
Empleada(o) doméstica & 2 & 1 & 3 & 6 & 7 & 13 & 8 & 8 & 16 \\
Otro no Pariente & 7 & 10 & 17 & 3 & 10 & 13 & 10 & 20 & 30 \\
Residente en vivienda colectiva & 1 & 3 & 4 & 1 & - & 1 & 2 & 3 \\
Total & 1523 & 338 & 1861 & 1618 & 369 & 1987 & 3141 & 707 & 3848 \\
\hline
\end{tabular}

Fuente: INE. Censo de Poblacion, 2001. [ http://www.ine.hn.org]

Del cuadro 3 se desprende que la estructura familiar es fuertemente nuclear y con un tamaño cercano a 6 miembros por vivienda, en la que predominan la presencia del padre, la madre y los hijos. Tal como ha sido reportado por evidencia etnológica (Lanza, 1992). 


\section{CENSO ETNO-DEMOGRÁFICO EN TERRITORIOS PECH 1994}

El estudio realizado por UDIP-IHAH, resulta ser el único trabajo específico sobre población étnica desde la perspectiva poblacional y con la particularidad de que, a su vez, buscó un acercamiento antropológico en cuanto características socio-culturales del grupo que ayudan a dar cuenta de su dinámica de población.

La boleta empleada en este estudio se organizo abarcando cuatro grandes secciones, los temas específicos fueron:

1. La vivienda y condiciones de sanidad (por comunidad)
- Localización y tenencia de la vivienda

- Tamaño, cantidad de cuartos y uso

- Abastecimiento del agua y

- Empleo de plantas medicinales

- Existencia de parteras tradicionales y lugar de nacimiento de los hijos

- Historia de hospitalizaciones

- Mortalidad de miembros de la vivienda (por sexo, edad, parentesco y fecha)

- Migración de miembros de la vivienda (por sexo, edad y fecha al partir, parentesco, tiempo de residir en la vivienda y lugar de destino -depto. Y municipio-)

2. Estructura familiar y - Edad y sexo

características demográficas (todas las personas residentes habituales en la vivienda)

- Estado civil

- Relación de parentesco

- Lugar de nacimiento (departamento y municipio)

- Fecha y razón de llegada al lugar

- Lengua que habla

- Alfabetismo, nivel educativo y asistencia a centro escolar

- Ocupación principal en la zona

- Fecha y ocupación fuera de la zona

- Total de hijos tenidos (nacidos vivos o muertos)

- Orden de nacimiento, sexo y fecha nacimiento

- Condición de sobre vivencia de los hijos

- Tamaño y tenencia de la unidad productiva

- Producción (producto, tiempo de producirlo, extensión sembrada, destino de la producción)

- Persona que decide la producción

- Herramientas y equipo empleados

- Insumos usados

- Condiciones de la comercialización 


\section{DATOS Y RESULTADOS DEL ESTUDIO ETNODEMOGRÁFICO DE 1994: APROXIMACIÓN.}

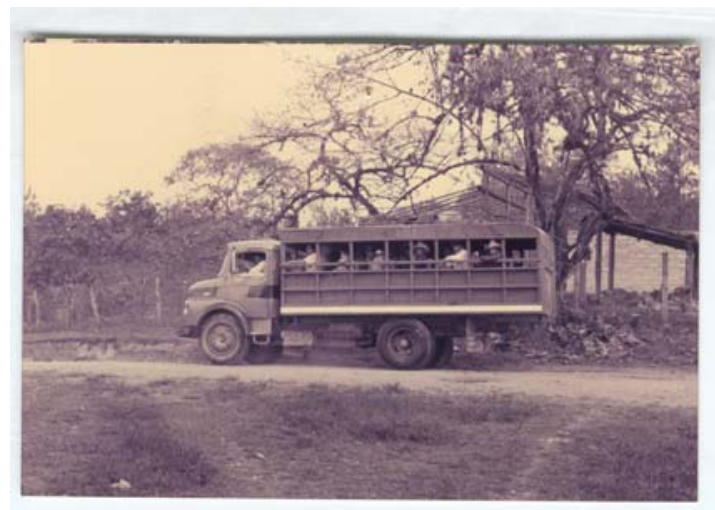

Los pech se definen a su mismos como la gente y se refieren al resto de la población como pech-akuá (la otra gente) o bulá (ladino), y rechazan la denominación que se hace de ellos como “payas o payitas” por que hace alusión a bárbaro, animal (Rivas, 1993).

El acceso a la mayoría de las comunidades debe hacerse por caminos de tierra, transitables en verano con pocas dificultades, pero en invierno se presentan fuertes limitaciones para su acceso. En "La Varonesa” (vehículo en la foto) se realiza el recorrido del poblado Nueva Subirana hasta Catacamas. Este último es cabecera del departamento de Olancho.

La población en los territorios pech y censada en 1994 alcanzo el volumen de 2079 personas, siendo un $49 \%$ hombres, como puede notarse en el cuadro 4.

\begin{tabular}{cccccc}
\multicolumn{6}{c}{ Cuadro 4. Población pech por sexo y viviendas según } \\
& $\begin{array}{c}\text { poblado.1994 } \\
\text { Hombre }\end{array}$ & Mujer & Total & Viviendas & Personas/ \\
\hline Nueva Subirana & 185 & 199 & 384 & 40 & 9.6 \\
Pisijire & 22 & 22 & 44 & 5 & 8.8 \\
Agua Zarcas & 46 & 45 & 91 & 12 & 7.6 \\
Jocomico & 27 & 32 & 59 & 7 & 8.4 \\
Culuco & 93 & 89 & 182 & 21 & 8.7 \\
Vallecito & 50 & 53 & 103 & 12 & 8.6 \\
El Carbón & 309 & 320 & 629 & 71 & 8.9 \\
Agua amarilla & 16 & 29 & 45 & 4 & 11.3 \\
Dulce nombre de Culmí & 19 & 16 & 35 & 3 & 11.7 \\
Coyolito & 28 & 30 & 58 & 7 & 8.3 \\
Zopilote & 32 & 38 & 70 & 4 & 17.5 \\
Silín & 73 & 64 & 137 & 19 & 7.2 \\
Las Marías & 120 & 122 & 242 & 35 & 6.9 \\
\hline Total & $\mathbf{1 0 2 0}$ & $\mathbf{1 0 5 9}$ & $\mathbf{2 0 7 9}$ & $\mathbf{2 4 0}$ & $\mathbf{8 . 7}$ \\
\hline Fuente: Censo Pech, 1994 & & & & &
\end{tabular}

Los poblados de El Carbón y Nueva Subirana, en el departamento de Olancho, son los que tienen la mayor cantidad de población. Las Marías, en La Mosquitia (departamento de Gracias a Dios) es el tercero. Los demás poblados tienen población escasa y en algunos se 
trata prácticamente de la misma familia, en varias viviendas, como se nota por la cantidad de personas por vivienda.

Cuadro 5. Población en poblados pech de 6 años y más por sexo según lengua que habla. 1994

\begin{tabular}{lccr}
\hline & Hombres & Mujeres & Total \\
Pech & 463 & 518 & 981 \\
Español & 246 & 203 & 449 \\
Otra & 53 & 67 & 120 \\
\hline
\end{tabular}

Fuente: Censo Pech, 1994

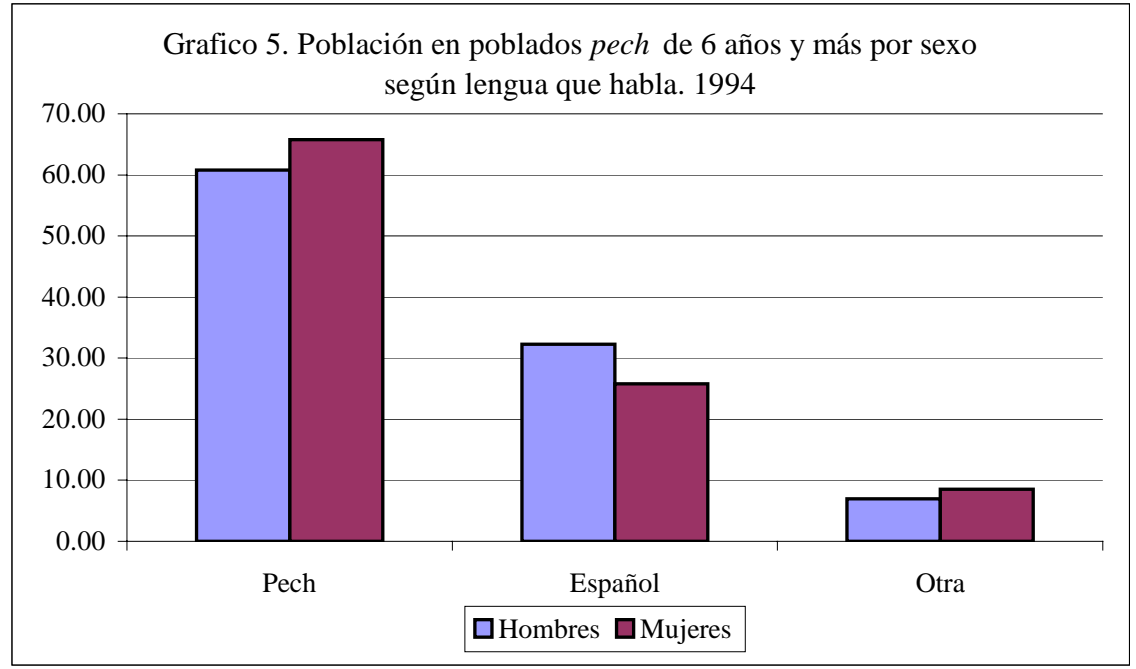

Fuente: Censo Pech, 1994

Como se indico, las mujeres son las que mas reportan hablar el pech o paya, y estas también son mayorías en el uso del español como segunda lengua (cuadro 6).

\begin{tabular}{cccc}
$\begin{array}{c}\text { Cuadro 6. Población en poblados pech de } 6 \text { años y } \\
\text { más por sexo según segunda lengua que habla. } \\
\end{array}$ & $\mathbf{1 9 9 4}$ & & \\
& Hombres & Mujeres & Total \\
\hline Lenca & & 0.26 & 0.13 \\
Misquito & 1.24 & 0.79 & 1.01 \\
Pech & 11.1 & 8.90 & 9.99 \\
Espanol & 68.6 & 75.0 & 71.9 \\
Ninguna & 19.0 & 15.1 & 17.0 \\
\hline
\end{tabular}

Fuente: Censo Pech, 1994

Pese a que una importante población pech conviven con población misquita en La Mosquitia (11\%), es muy poco el manejos de la lengua misquita que tienen (1\%). Como se había comentado, desde tiempos de la Colonia, los misquitos mostraron tener manejo de las 
lenguas para poder comerciar. Así, posiblemente no ha sido “necesario” para la población pech el aprender esta lengua, pues los misquitos mas bien hablan el pech o se comunican en español.

\section{Actividades productivas}

Caza y pesca

La actividad de la caza, de especies menores especialmente, ha sido importante y tradicional en la cultura pech. Pájaros,

Desde muy temprana edad, los niños conocen sobre el arte de la caza. El niño en la foto muestra su "rifle", de auto fabricación y cuyo mecanismo es accionado por una palanca que libera una "liga” la cual deja escapar la lanza.
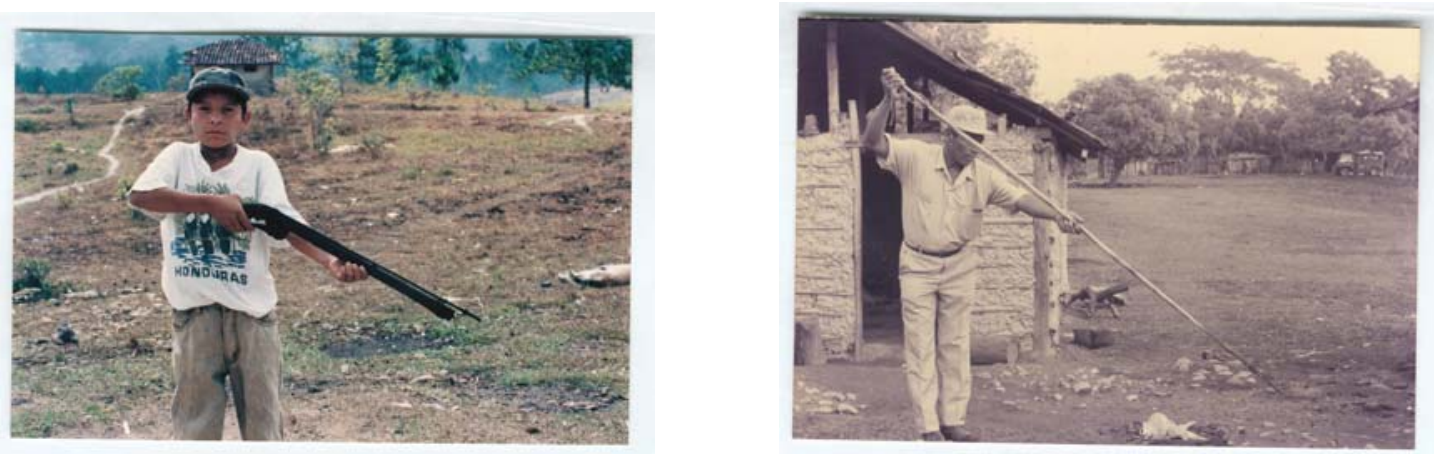

\section{Granos básicos.}

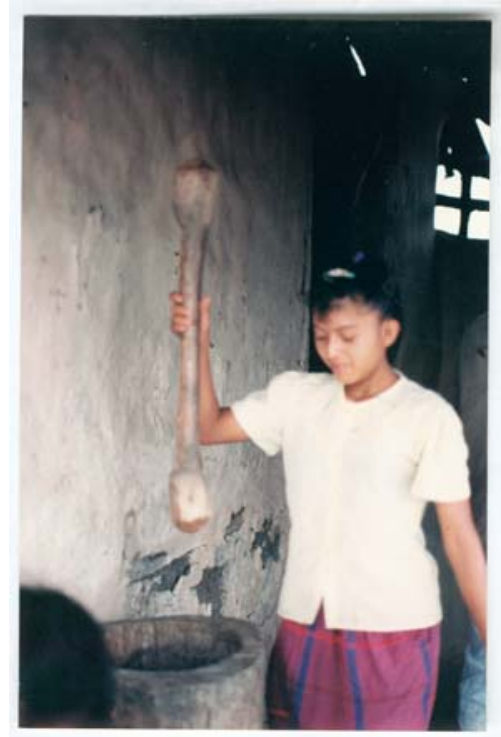

En esta grafica, la muchacha emplea el “pilon” para preparar el arroz, quitándole la cáscara, para poder preparar la comida. Es común que la preparación del arroz se realice según las necesidades, y no como sucede en contextos "ladinos" en que se descascara el arroz de toda la cosecha de una vez.

El maíz es el principal producto (41\%), seguido de la yuca y tubérculos (20\%), el arroz (10\%) y los frijoles (7\%). Esto evidencia la persistencia de las raíces como cultivo tradicional e importante en su dieta. 


\section{La vivienda}

Como se indico, la población pech se distribuye en 14 poblados, y en 240 viviendas, en su mayoría organizadas en poblados "concentrados". La residencia es matrilocal ${ }^{5}$

Del total de viviendas, el $22 \%$ consta tan solo de un aposento y $44 \%$ tiene dos, que normalmente son uno como dormitorio y el otro es para diversos usos.

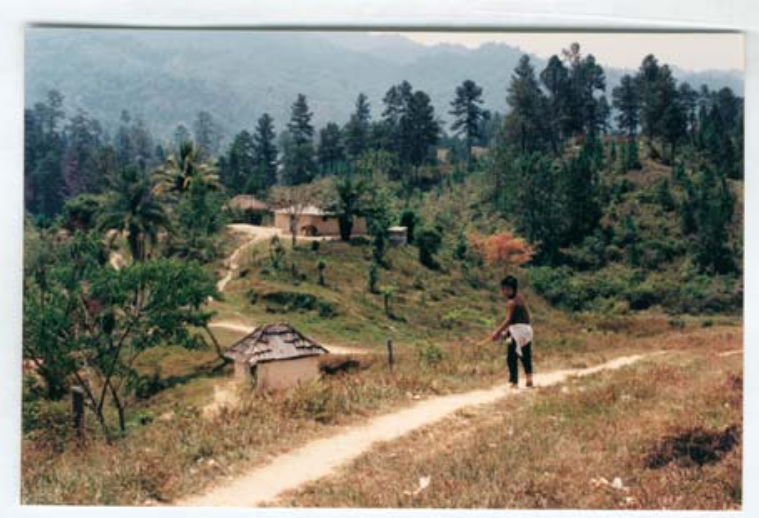

En el 75\% de los casos el ocote (un árbol de pino muy resinoso) es lo que emplean para alumbrarse, seguido de un $20 \%$ por medio de gas licuado (LPG). Como combustible, en el 95\% se emplea la leña

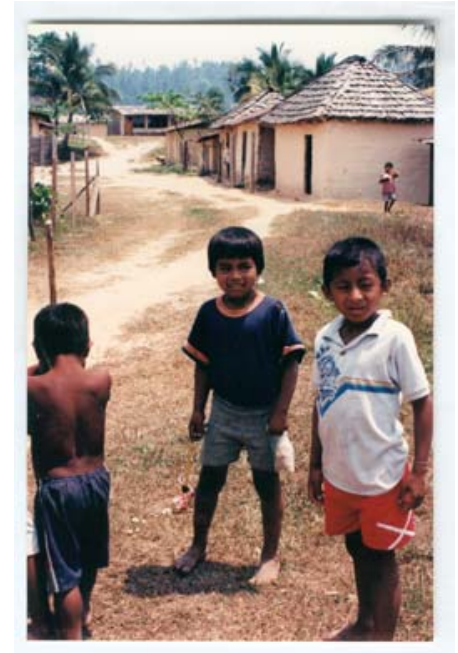

Consultados sobre el empleo de plantas medicinales, en el 79\% de las viviendas hacían uso "frecuente" de estas. En el $97 \%$ se reconoce la existencia de parteras, auque su determinación es muy variada: desde 3 hasta 40, siendo 2 el numero mas mencionado. Y en el 97\% de los casos, reportaron haber tenido sus hijos en la vivienda, en su mayoría asistidas por la partera.

En el 29\% de las viviendas se reporto algún miembro había emigrado, siendo el 62\% de estos hijo del jefe. Tanto hombres como mujeres han emigrado en igual proporción (50\%).

\footnotetext{
${ }^{5}$ Cuando se casan (o unen), el "yerno” se traslada a vivir al poblado de su compañera, y en algunos casos a la misma vivienda. Con ello se aumenta la fuerza de trabajo de la familia de la novia.
} 


\section{CONCLUSIONES}

La situación descrita y las lecciones que arrojan los estudios aquí comentados apuntan en la necesidad de que dentro de los estudios de población se realicen esfuerzos para particularizar en la realidad socio-demográfica de los pueblos étnicos de Honduras. Esta tarea debería ser realizada a la brevedad y con el concurso no sólo de especialistas en población, sino, por la complejidad que estas poblaciones presentan, debe realizarse en forma interdisciplinaria más que multidisciplinaria.

Si bien un alto porcentaje de la población aun habla el pech, su perdida y la penetración del español hacen que un importante identificador de la etnia este amenazando la sobrevivencia del grupo como grupo étnico. Aunque el aun importante empleo de raíces en su dieta y en el cultivo, muestran fortalezas para la sobrevivencia de la cultura. Estas estan muy relacionadas con bebidas tradicionales y ceremonias, siendo otro de los importantes ejes de la cultura.

El determinar y estudiar las poblaciones étnicas es una labor compleja y con diversas dificultades. Se debe partir de una definición que permita clarificar la población. Se han ensayado cuatro posibilidades: la raza o fenotipo, la lengua o idioma empleado, la presencia en los territorios étnicos y la "auto adscripción". Ninguna por si sola permite dar cuenta clara de esta población.

Consideramos que para una adecuada determinación de las poblaciones étnicas, no bastara con una definición "previa” ni con la auto descripción, se deberá realizar mediante una mezcla de ambas, para dar mayor riqueza y dinamismo a la definición. Los criterios de la primera deberán estar enfocados al empleo de la lengua o idioma y variables de la cultura (costumbres, ritos, y similares) y en ningún caso sujetos a la definición "racial” y menos por el fenotipo. 


\section{BIBLIOGRAFÍA}

Benzoi, G. (1967). La historia del Nuevo Mundo. Carácas, Academia Nacional de la Historia (reedición del original de 1541).

CELADE. (1994). Estudios sociodemográficos de pueblos indígenas. Serie E, $\mathrm{N}^{\circ} 40$. Santiago de Chile: CELADE/CIDOB/FNUAP/ICI.

Cruz Sandoval, Fernando. (1984). "Los indios de Honduras y la situación de sus recursos naturales”. América Indígena. 3 (XLIV). México: Instituto Indigenista Interamericano.

DGEC. Censos de Población (varios años) . Tegucigalpa: DGEC.

Dobyns, H. F. (1966). "Estimating aboriginal American population". En: Current Anthropology 7: 395-449.

Griffin, Wendy (1992). Historia de los indígenas de la zona nororiental de Honduras. Tegucigalpa, Universidad Pedagógica Nacional (2 vols.).

INEC-Honduras. (2003). Censo de población. [ http://www.INEC.org.hn/ ]

Keper, Charles D. Jr. (1936). Social aspects of the banana industry. New York, Columbia Univer-sity Press.

Kroeber, A. L. (1939). Cultural and natural areas of native North America. Berkeley, University of California Press, Publication in Archaeology and Ethnology 38.

Lanza, Roberto de J. et al. (1992). Los pech (payas): una cultura olvidada. 2da Ed. Honduras: Editorial Guaymuras.

MacLead, Murdo (1973). Spanish Central America: a socio-economic history 1520-1720. Berkeley, University of California Press.

Mariñas, OTERO, Luis (1987). Honduras. Tegucigalpa, Editorial Universitaria.

Newson, Linda. (1981). El costo de la conquista. Tegucigalpa: Editorial Guaymuras.

PAH. (1989). Perfil ambiental de Honduras. Washington DC, SECPLAN/DESFIL.

Peyser Alexia y Juan Chackiel. (1994). "La población indígena en los censos de America Latina” . En: Estudios sociodemográficos de pueblos indígenas. Santiago, Chile: CELADE/CIDOB/FNUAP/ICI.

Ponce, Fernando (1993) b. Pueblos indígenas y tribales en Honduras. Exploración de las características poblacionales, socioeconómicas y de pobreza y potencialidad por Departamento a partir del Censo Nacional de Población de 1988. Tegucigalpa, SECPLAN, Proyecto HON/ 90/P03. 
Rivas, Ramón D. (1993). Pueblos indígenas y garífuna de Honduras (una caracterización). Honduras: Servicio holandés de cooperación al Desarrollo/Editorial Guaymuras.

Sapper, K. (1924). "Die Zahl und die Volksdichte der Indianischen Bevölkerung in Amerika vor der Conquista und in der Gegenwart". En: 21st International Congress of Americanists, The Hague 1: 95-104.

Squier, E. George (1856). Apuntamiento sobre Centroamérica. Particularmente sobre los Estados de Honduras y El Salvador. Paris, Imp. Gustavo Gratior (traducción de original en inglés publicado en 1855).

Tamayo, Patricia (1994). Diagnóstico de la información socio-demográfica existente respecto al grupo étnico Tawahka-Sumo de Honduras. Tegucigalpa, UDIP-UNAH.

Tojiera, José María (1982). Los jicaques de Yoro. Tegucigalpa, Guaymuras.

Valdes, Luz María. (1988). Perfil demográfico de los indios mexicanos. México: Siglo XXI.

Vallejo, Antonio R. (1893). Primer Anuario Estadístico de la República de Honduras, correspondiente al año de 1889. Tegucigalpa, Tipografía Nacional.

Wells, William (1982). Exploraciones y aventuras en Honduras. Guatemala, Editorial Universitaria Centroamericana. 